BULLETIN (New Series) OF THE

AMERICAN MATHEMATICAL SOCIETY

Volume 44, Number 1, January 2007, Pages 1-18

S 0273-0979(06)01142-6

Article electronically published on September 25, 2006

\title{
SMALL GAPS BETWEEN PRIME NUMBERS: THE WORK OF GOLDSTON-PINTZ-YILDIRIM
}

\author{
K. SOUNDARARAJAN
}

\section{INTRODUCTION}

In early 2005, Dan Goldston, János Pintz, and Cem Yıldırım [12] made a spectacular breakthrough in the study of prime numbers. Resolving a long-standing open problem, they proved that there are infinitely many primes for which the gap to the next prime is as small as we want compared to the average gap between consecutive primes. Before their work, it was known only that there were infinitely many gaps which were about a quarter the size of the average gap. The new result may be viewed as a step towards the famous twin prime conjecture that there are infinitely many prime pairs $p$ and $p+2$, the gap here being 2 , the smallest possible gap between primes 11 Perhaps most excitingly, their work reveals a connection between the distribution of primes in arithmetic progressions and small gaps between primes. Assuming certain (admittedly difficult) conjectures on the distribution of primes in arithmetic progressions, they are able to prove the existence of infinitely many prime pairs that differ by at most 16 . The aim of this article is to explain some of the ideas involved in their work.

Let us begin by explaining the main question in a little more detail. The number of primes up to $x$, denoted by $\pi(x)$, is roughly $x / \log x$ for large values of $x$; this is the celebrated Prime Number Theorem 2 Therefore, if we randomly choose an integer near $x$, then it has about a $1-\operatorname{in}-\log x$ chance of being prime. In other words, as we look at primes around size $x$, the average gap between consecutive primes is about $\log x$. As $x$ increases, the primes get sparser and the gap between consecutive primes tends to increase. Here are some natural questions about these gaps between prime numbers. Do the gaps always remain roughly about size $\log x$, or do we sometimes get unexpectedly large gaps and sometimes surprisingly small gaps? Can we say something about the statistical distribution of these gaps? That is, can we quantify how often the gap is between, say, $\alpha \log x$ and $\beta \log x$, given $0 \leq \alpha<\beta$ ? Except for the primes 2 and 3, clearly the gap between consecutive primes must be even. Does every even number occur infinitely often as a gap between consecutive primes? For example, the twin prime conjecture says that the

Received by the editors July 18, 2006.

2000 Mathematics Subject Classification. Primary 11N05.

This article is based on a lecture presented January 14, 2006, at the AMS Special Session on Current Events, Joint Mathematics Meetings, San Antonio, TX.

The author is partially supported by the National Science Foundation.

${ }^{1}$ Apart from the gap between 2 and 3 , of course!

${ }^{2}$ Here, and throughout, log stands for the natural logarithm.

(C)2006 American Mathematical Society Reverts to public domain 28 years from publication 
gap 2 occurs infinitely. How frequently should we expect the occurrence of twin primes?

Number theorists believe they know the answers to all these questions but cannot always prove that the answers are correct. Before discussing the answers let us address a possible meta-question. Problems like twin primes and the Goldbach conjecture involve adding and subtracting primes. The reader may well wonder if such questions are natural or just isolated curiosities. After all, shouldn't we be multiplying with primes rather than adding/subtracting them? There are several possible responses to this objection.

Firstly, many number theorists and mathematical physicists are interested in understanding spacing statistics of various sequences of numbers occurring in nature. Examples of such sequences are prime numbers, the ordinates of zeros of the Riemann zeta-function (see 21] and 23]), energy levels of large nuclei, the fractional parts of $\sqrt{n}$ for $n \leq N$ (see [7]), etc. Do the spacings behave like the gaps between randomly chosen numbers, or do they follow more esoteric laws? Our questions on gaps between primes fit naturally into this framework.

Secondly, many additive questions on primes have applications to other problems in number theory. For example, consider primes $p$ for which $2 p+1$ is also a prime. Analogously to twin primes, it is conjectured that there are infinitely many such prime pairs $p$ and $2 p+1$. Sophie Germain came up with these pairs in her work on Fermat's last theorem. If there are infinitely many Germain pairs $p$ and $2 p+1$ with $p$ lying in a prescribed arithmetic progression, then Artin's primitive root conjecture - every positive number $a$ which is not a perfect square is a primitive root 3 for infinitely many primes - would follow. For example, if $p$ lies in the progression $3(\bmod 40)$, and $2 p+1$ is prime, then 10 is a primitive root modulo $2 p+1$, and as Gauss noticed (and the reader can check) the decimal expansion of $1 /(2 p+1)$ has exactly $2 p$ digits that repeat. There are also connections between additive questions on primes and zeros of the Riemann zeta and other related functions. Precise knowledge of the frequency with which prime pairs $p$ and $p+2 k$ occur (for an even number $2 k$ ) has subtle implications for the distribution of spacings between ordinates of zeros of the Riemann zeta-function (see [1] and [23]). Going in the other direction, weird (and unlikely) patterns in zeros of zeta-like functions would imply the existence of infinitely many twin primes (see [17])!

Finally, these 'additive' questions on primes are lots of fun, have led to much beautiful mathematics, and have inspired many generations of number theorists!

Cramér's model. A useful way to think about statistical questions on prime numbers is the random - also known as Cramér - model. The principle, based on the fact that a number of size about $n$ has a 1 in $\log n$ chance of being prime, is this:

The indicator function for the set of primes (that is, the function whose value at $n$ is 1 or 0 depending on whether $n$ is prime or not) behaves roughly like a sequence of independent, Bernoulli random variables $X(n)$ with parameters $1 / \log n(n \geq 3)$. In other words, for $n \geq 3$, the random variable $X(n)$ takes the value 1 ( $n$ is 'prime') with probability $1 / \log n$, and $X(n)$ takes the value 0 ( $n$ is 'composite') with probability $1-1 / \log n$. For completeness, let us set $X(1)=0$ and $X(2)=1$.

\footnotetext{
${ }^{3}$ That is, $a$ generates the multiplicative group of residues modulo that prime.
} 
This must be taken with a liberal dose of salt: a number is either prime or composite; probability does not enter the picture! Nevertheless, the Cramér model is very effective in predicting answers, although it does have its limitations (for example, if $n>2$ is prime, then certainly $n+1$ is not, so the events of $n$ and $n+1$ being prime are clearly not independent) and sometimes leads to incorrect predictions.

Let us use the Cramér model to predict the probability that, given a large prime $p$, the next prime lies somewhere between $p+\alpha \log p$ and $p+\beta \log p$. In the Cramér model, let $p$ be large and suppose that $X(p)=1$. What is the probability that $X(p+1)=X(p+2)=\ldots=X(p+h-1)=0$ and $X(p+h)=1$, for some integer $h$ in the interval $[\alpha \log p, \beta \log p]$ ? We will find this by calculating the desired probability for a given $h$ in that interval and summing that answer over all such $h$. For a given $h$ the probability we seek is

$$
\left(1-\frac{1}{\log (p+1)}\right)\left(1-\frac{1}{\log (p+2)}\right) \cdots\left(1-\frac{1}{\log (p+h-1)}\right) \frac{1}{\log (p+h)} .
$$

Since $p$ is large and $h$ is small compared to $p$ (it's only of size about $\log p$ ), we estimate that $\log (p+j)$ is very nearly $\log p$ for $j$ between 1 and $h$. Therefore our probability above is approximately $(1-1 / \log p)^{h-1}(1 / \log p)$, and since $1-1 / \log p$ is about $e^{-1 / \log p}$, this is roughly

$$
e^{-(h-1) / \log p}\left(\frac{1}{\log p}\right) .
$$

Summing over the appropriate $h$, we find that the random model prediction for the probability that the next prime larger than $p$ lies in $[p+\alpha \log p, p+\beta \log p]$ is

$$
\sum_{\alpha \log p \leq h \leq \beta \log p} e^{-(h-1) / \log p} \frac{1}{\log p} \approx \int_{\alpha}^{\beta} e^{-t} d t
$$

since the left-hand side looks like a Riemann sum approximation to the integral.

Conjecture 1. Given an interval $0 \leq \alpha<\beta$, as $x \rightarrow \infty$ we have

$$
\frac{1}{\pi(x)} \#\left\{p \leq x: p_{\text {next }} \in(p+\alpha \log p, p+\beta \log p)\right\} \rightarrow \int_{\alpha}^{\beta} e^{-t} d t
$$

where $p_{\text {next }}$ denotes the next prime larger than $p$. Here and throughout the paper, the letter $p$ is reserved for primes.

We have deliberately left the integral unevaluated to suggest that there is a probability density $e^{-t}$ of finding $\left(p_{\text {next }}-p\right) / \log p$ close to $t$. If we pick $N$ random numbers uniformly and independently from the interval $[0, N]$ and arrange them in ascending order, then, almost surely, the consecutive spacings have the probability density $e^{-t}$. Thus, the Cramér model indicates that the gaps between consecutive primes are distributed like the gaps between roughly $x / \log x$ numbers chosen uniformly and independently from the interval $[0, x]$. In probability terminology, this is an example of what is known as a 'Poisson process'.

There are several related predictions we could make using the random model. For example, choose a random number $n$ below $x$ and consider the interval $[n, n+\log n]$. The expected number of primes in such an interval is about 1 , by the prime number theorem. But of course some intervals may contain no prime at all while others may contain several primes. Given a non-negative number $k$, what is the probability that such an interval contains exactly $k$ primes? The reader may enjoy the pleasant 
calculation which predicts that, for large $x$, the answer is nearly $\frac{1^{k}}{k !} e^{-1}$ - the answer is written so as to suggest a Poisson distribution with parameter 1 .

Conjecture 1 makes clear that there is substantial variation in the gaps between consecutive primes. Given any large number $\Lambda$, we expect that with probability about $e^{-\Lambda}$ (a tiny but positive probability), the gap between consecutive primes is more than $\Lambda$ times the average gap. Given any small positive number $\epsilon$, we expect that with probability about $1-e^{-\epsilon}$ (a small but positive probability), the gap between consecutive primes is at most $\epsilon$ times the usual gap. Thus, two consequences of Conjecture 1 are

$$
\limsup _{p \rightarrow \infty} \frac{p_{\text {next }}-p}{\log p}=\infty
$$

and

$$
\liminf _{p \rightarrow \infty} \frac{p_{\text {next }}-p}{\log p}=0
$$

Large gaps. Everyone knows how to construct arbitrarily long intervals of composite numbers: just look at $m !+2, m !+3, \ldots, m !+m$ for any natural number $m \geq 2$. This shows that $\lim \sup _{p \rightarrow \infty}\left(p_{\text {next }}-p\right)=\infty$. However, if we think of $m$ ! being of size about $x$, then a little calculation with Stirling's formula shows that $m$ is about size $(\log x) / \log \log x$. We realize, with dismay, that the 'long' gap we have constructed is not even as large as the average gap of $\log x$ given by the prime number theorem. A better strategy is to take $N$ to be the product of the primes that are at most $m$, and note again that $N+2, \ldots, N+m$ must all be composite. It can be shown that $N$ is roughly of size $e^{m}$. Thus we have found a gap at least of size $\log N$, which is better than before, but still not better than average. Can we modify the argument a little? In creating our string of $m-1$ consecutive composite numbers, we forced these numbers to be divisible by some prime below $m$. Can we somehow use primes larger than $m$ to force $N+m+1, N+m+2$, etc., to be composite and thus create longer chains of composite numbers? In the 1930s, in a series of papers Westzynthius [27, Erdös [8] and Rankin [25] found ingenious ways of making this idea work. The best estimate was obtained by Rankin, who proved that there exists a positive constant $c$ such that for infinitely many primes $p$,

$$
p_{\text {next }}-p>c \log p \frac{(\log \log p) \log \log \log \log p}{(\log \log \log p)^{2}} \text {. }
$$

The fraction above does grow 4 and so

$$
\limsup _{p \rightarrow \infty} \frac{p_{\text {next }}-p}{\log p}=\infty
$$

as desired. We should remark here that, although very interesting work has been done on improving the constant $c$ above, Rankin's result provides the largest known gap between primes. Erdős offered $\$ 10,000$ for a similar conclusion involving a faster growing function. Bounty hunters may note that the largest Erdös prize that has been collected is $\$ 1,000$, by Szemeredi [26] for his marvellous result on the existence of long arithmetic progressions in sets of positive density.

\footnotetext{
${ }^{4}$ Although so slowly that, as the joke goes, no one has observed it doing so!
} 
What should we conjecture for the longest gap between primes? Cramér's model suggests that

$$
\limsup _{p \rightarrow \infty} \frac{p_{\text {next }}-p}{(\log p)^{2}}=c,
$$

with $c=1$. The rationale behind this is that the probability that $X(n)=1$ and that the next 'prime' is bigger than $n+(1+\epsilon) \log ^{2} n$ is about $1 /\left(n^{1+\epsilon} \log n\right)$, by a calculation similar to the one leading up to Conjecture 1. If $\epsilon$ is negative, the sum of this probability over all $n$ diverges and the Borel-Cantelli lemma tells us that almost surely such long gaps occur infinitely often. If $\epsilon$ is positive, the corresponding sum converges and the Borel-Cantelli lemma says that almost surely we get these longer gaps only a finite number of times. More sophisticated analysis has, however, revealed that (1) is one of those questions which expose the limitations of the Cramér model. It appears unlikely that the value of $c$ is 1 as predicted by the Cramér model and that $c$ should be at least $2 e^{-\gamma} \approx 1.1229$ where $\gamma$ is Euler's constant. No one has felt brave enough to suggest what the precise value of $c$ should be! This is because (1) is far beyond what 'reasonable' conjectures such as the Riemann hypothesis would imply. An old conjecture says that there is always a prime between two consecutive squares. Even this lies (slightly) beyond the reach of the Riemann hypothesis, and all it would imply is that

$$
\limsup _{p \rightarrow \infty} \frac{p_{\text {next }}-p}{\sqrt{p}} \leq 4
$$

a statement much weaker than (1) with a finite value of $c$.

We cut short our discussion on long gaps here, since our focus will be on small gaps; for more information on these and related problems, we refer the reader to the excellent survey articles by Heath-Brown [18] and Granville [15.

Small gaps. Since the average spacing between $p$ and $p_{\text {next }}$ is about $\log p$, clearly

$$
\liminf _{p \rightarrow \infty} \frac{p_{\text {next }}-p}{\log p} \leq 1
$$

Erdös [9] was the first to show that the lim inf is strictly less than 1 . Other landmark results in the area are the works of Bombieri and Davenport [3, Huxley [20, and Maier [22, who introduced several new ideas to this study and progressively reduced the liminf to $\leq 0.24 \ldots$ Enter Goldston, Pintz, and Yıldırım:

Theorem 1. We have

$$
\liminf _{p \rightarrow \infty} \frac{p_{\text {next }}-p}{\log p}=0 .
$$

So there are substantially smaller gaps between primes than the average! What about even smaller gaps? Can we show that $\liminf _{p \rightarrow \infty}\left(p_{\text {next }}-p\right)<\infty$ (bounded gaps), or perhaps even $\liminf _{p \rightarrow \infty}\left(p_{\text {next }}-p\right)=2$ (twin primes!)?

Theorem 2. Suppose the Elliott-Halberstam conjecture on the distribution of primes in arithmetic progressions holds true. Then

$$
\liminf _{p \rightarrow \infty}\left(p_{\text {next }}-p\right) \leq 16 .
$$

What is the Elliott-Halberstam conjecture? Vaguely, the Goldston-PintzYlldirım results say that if the primes are well separated with no small gaps between them, then something weird must happen to their distribution in arithmetic 
progressions. We seek to dismiss that possibility by understanding the distribution of primes in arithmetic progressions. For the proof of Theorem 1, the BombieriVinogradov theorem provides the necessary information. To obtain the stronger conclusion of Theorem 2, one requires a finer understanding of the distribution of primes in progressions, and this is where the Elliott-Halberstam conjecture comes in.

Given a progression $a(\bmod q)$ let $\pi(x ; q, a)$ denote the number of primes below $x$ lying in this progression. Naturally we may suppose that $a$ and $q$ are coprime; else there is at most one prime in the progression. Now there are $\phi(q)$ - this is Euler's $\phi$-function - such progressions $a(\bmod q)$ with $a$ coprime to $q$. We would expect that each progression captures its fair share of primes. In other words we expect that $\pi(x ; q, a)$ is roughly $\pi(x) / \phi(q)$. The prime number theorem in arithmetic progressions tells us that this is true if we view $q$ as being fixed and let $x$ go to infinity.

In applications such as Theorem 1, we need information on $\pi(x ; q, a)$ when $q$ is not fixed, but growing with $x$. When $q$ is growing slowly, say $q$ is $\operatorname{like} \log x$, the prime number theorem in arithmetic progressions still applies. However, if $q$ is a little larger, say $q$ is of size $x^{\frac{1}{3}}$, then currently we cannot prove the equidistribution of primes in the available residue classes $(\bmod q)$. Such a result would be implied by the Generalized Riemann Hypothesis (indeed for $q$ up to about $\sqrt{x}$ ), but of course the Generalized Riemann Hypothesis remains unresolved. In this context, Bombieri and Vinogradov showed that the equidistribution of primes in progressions holds, not for each individual $q$, but on average over $q$ (that is, for a typical $q$ ) for $q$ going up to about $\sqrt{x}$. Their result may be thought of as the 'Generalized Riemann Hypothesis on average'.

The Elliott-Halberstam conjecture says that the equidistribution of primes in progressions continues to hold on average for $q$ going up to $x^{1-\epsilon}$ for any given positive $\epsilon$. In some ways, this lies deeper than the Generalized Riemann Hypothesis, which permits only $q \leq \sqrt{x}$.

We hope that the reader has formed a rough impression of the nature of the assumption in Theorem 2. We will state the Bombieri-Vinogradov theorem and Elliott-Halberstam conjecture precisely in the penultimate section devoted to primes in progressions.

The Hardy-Littlewood conjectures. We already noticed a faulty feature of the Cramér model: given a large prime $p$, the probability that $p+1$ is prime is not $1 / \log (p+1)$ but 0 because $p+1$ is even. Neither would we expect the conditional probability of $p+2$ being prime to be simply $1 / \log (p+2)$ : after all, $p+2$ is guaranteed to be odd, and this should give it a better chance of being prime. How should we formulate the correct probability for $p+2$ being prime? More precisely, what should be the conjectural asymptotics for

$$
\#\{p \leq x: p+2 \text { prime }\} \text { ? }
$$

The Cramér model would have predicted that this is about $x /(\log x)^{2}$. While we must definitely modify this, it also seems reasonable that $x /(\log x)^{2}$ is the right size for the answer. So maybe the answer is about $c x /(\log x)^{2}$ for an appropriate constant $c$.

Long ago Hardy and Littlewood [16] figured out what the right conjecture should be. The problem with the Cramér model is that it treats $n$ and $n+2$ as being 
independent, whereas they are clearly dependent. If we want $n$ and $n+2$ both to be prime, then they must both be odd, neither of them must be divisible by 3 nor by 5 , and so on. If we choose $n$ randomly, the probability that $n$ and $n+2$ are both odd is $1 / 2$. In contrast, two randomly chosen numbers would both be odd with a $1 / 4$ probability. If neither $n$ nor $n+2$ is divisible by 3 , then $n$ must be $2(\bmod 3)$, which has a $1 / 3$ probability. On the other hand, the probability that two randomly chosen numbers are not divisible by 3 is $(2 / 3) \cdot(2 / 3)=4 / 9$. Similarly, for any prime $\ell \geq 3$, the probability that $n$ and $n+2$ are not divisible by $\ell$ is $1-2 / \ell$, which is a little different from the probability $(1-1 / \ell)^{2}$ that two randomly chosen integers are both not divisible by $\ell$. For the prime 2 we must correct the probability $1 / 4$ by multiplying by $2=(1-1 / 2)(1-1 / 2)^{-2}$, and for all primes $\ell \geq 3$ we must correct the probability $(1-1 / \ell)^{2}$ by multiplying by $(1-2 / \ell)(1-1 / \ell)^{-2}$. The idea is that if we multiply all these correction factors together then we have accounted for 'all the ways' in which $n$ and $n+2$ are dependent, producing the required correction constant $c$. Thus the conjectured value for $c$ is the product over primes

$$
\left(1-\frac{1}{2}\right)\left(1-\frac{1}{2}\right)^{-2} \prod_{\ell \geq 3}\left(1-\frac{2}{\ell}\right)\left(1-\frac{1}{\ell}\right)^{-2} \text {. }
$$

Let us make a synthesis of the argument above, which will allow us to generalize it. For any prime $\ell$ let $\nu_{\{0,2\}}(\ell)$ denote the number of distinct residue classes $(\bmod \ell)$ occupied by the numbers 0 and 2. If we want both $n$ and $n+2$ to be coprime to $\ell$, then $n$ must avoid the residue classes occupied by -0 and $-2(\bmod \ell)$, so that $n$ must lie in one of $\ell-\nu_{\{0,2\}}(\ell)$ residue classes. The probability that this happens is $1-\nu_{\{0,2\}}(\ell) / \ell$, so the correction factor for $\ell$ is $\left(1-\nu_{\{0,2\}}(\ell) / \ell\right)(1-1 / \ell)^{-2}$. As before, consider the infinite product over primes

$$
\mathfrak{S}(\{0,2\}):=\prod_{\ell}\left(1-\frac{\nu_{\{0,2\}}(\ell)}{\ell}\right)\left(1-\frac{1}{\ell}\right)^{-2} .
$$

The infinite product certainly converges: the terms for $\ell \geq 3$ are all less than 1 in size. Moreover, it converges to a non-zero number. Note that none of the factors above is zero and that for large $\ell$ the logarithm of the corresponding factor above is very small: it is $\log \left(1-1 /(\ell-1)^{2}\right) \approx-1 / \ell^{2}$. Thus the sum of the logarithms converges, and the product is non-zero; indeed $\mathfrak{S}(\{0,2\})$ is numerically about 1.3203. Then the conjecture is that for large $x$

$$
\#\{p \leq x: p+2 \text { prime }\} \sim \mathfrak{S}(\{0,2\}) \frac{x}{(\log x)^{2}} .
$$

Here and below, the notation $f(x) \sim g(x)$ means that $\lim _{x \rightarrow \infty} f(x) / g(x)=1$.

The conjecture generalizes readily: Suppose we are given a set $\mathcal{H}=\left\{h_{1}, h_{2}, \ldots\right.$, $\left.h_{k}\right\}$ of non-negative integers and we want to find the frequency with which $n+h_{1}$, $\ldots, n+h_{k}$ are all prime. For a prime number $\ell$, we define $\nu_{\mathcal{H}}(\ell)$ to be the number of distinct residue classes $(\bmod \ell)$ occupied by $\mathcal{H}$. We define the 'singular series 5

$$
\mathfrak{S}(\mathcal{H})=\prod_{\ell}\left(1-\frac{\nu_{\mathcal{H}}(\ell)}{\ell}\right)\left(1-\frac{1}{\ell}\right)^{-k}
$$

\footnotetext{
${ }^{5}$ The terminology is not entirely whimsical: Hardy and Littlewood originally arrived at their conjecture through a heuristic application of their 'circle method'. In their derivation, $\mathfrak{S}(\mathcal{H})$ did arise as a series rather than as our product.
} 
If $\ell$ is larger than all elements of $\mathcal{H}$, then $\nu_{\mathcal{H}}(\ell)=k$, and for such $\ell$ the terms in the product are less than 1 . Thus the product converges. When does it converge to a non-zero number? If $\nu_{\mathcal{H}}(\ell)=\ell$ for some prime $\ell$, then one of the terms in our product vanishes, and so our product must be zero. Suppose none of the terms is zero. For large $\ell$ the logarithm of the corresponding factor is

$$
\log \left(1-\frac{k}{\ell}\right)\left(1-\frac{1}{\ell}\right)^{-k} \approx-\frac{k(k+1)}{2 \ell^{2}}
$$

and so the sum of the logarithms converges and our product is non-zero. Thus the singular series is zero if and only if $\nu_{\mathcal{H}}(\ell)=\ell$ for some prime $\ell$ - that is, if and only if the numbers $h_{1}, \ldots, h_{k}$ occupy all the residue classes $(\bmod \ell)$ for some prime $\ell$. In that case, for any $n$ one of the numbers $n+h_{1}, \ldots, n+h_{k}$ must be a multiple of $\ell$, and so there are only finitely many prime $k$-tuples $n+h_{1}, \ldots, n+h_{k}$.

The Hardy-Littlewood conjecture. Let $\mathcal{H}=\left\{h_{1}, \ldots, h_{k}\right\}$ be a set of positive integers such that $\mathfrak{S}(\mathcal{H}) \neq 0$. Then

$$
\#\left\{n \leq x: n+h_{1}, \ldots, n+h_{k} \text { prime }\right\} \sim \mathfrak{S}(\mathcal{H}) \frac{x}{(\log x)^{k}}
$$

It is easy to see that $\mathfrak{S}(\{0,2 r\}) \neq 0$ for every non-zero even number $2 r$. Thus the Hardy-Littlewood conjecture predicts that there are about $\mathfrak{S}(\{0,2 r\}) x /(\log x)^{2}$ prime pairs $p$ and $p+2 r$ with $p$ below $x$. Further, the number of these pairs for which $p+2 d$ is also prime for some $d$ between 1 and $r-1$ is at most a constant times $x /(\log x)^{3}$. We deduce that there should be infinitely many primes $p$ for which the gap to the next prime is exactly $2 r$. Thus every positive even number should occur infinitely often as a gap between successive primes, but we don't know this for a single even number!

For any $k$, it is easy to find $k$-element sets $\mathcal{H}$ with $\mathfrak{S}(\mathcal{H}) \neq 0$. For example, take $\mathcal{H}$ to be any $k$ primes all larger than $k$. Clearly if $\ell>k$, then $\nu_{\mathcal{H}}(\ell) \leq k<\ell$, while if $\ell \leq k$, then the residue class $0(\bmod \ell)$ must be omitted by the elements of $\mathcal{H}$ (they are primes!) and so once again $\nu_{\mathcal{H}}(\ell)<\ell$.

We make one final comment before turning (at last!) to the ideas behind the proofs of Theorems 1 and 2. Conjecture 1 was made on the strength of the Cramér model, but we have just been discussing how to modify the Cramér probabilities for prime $k$-tuples. A natural question is whether the Hardy-Littlewood conjectures are consistent with Conjecture 1. In a beautiful calculation [11, Gallagher showed that Conjecture 1 can in fact be obtained starting from the Hardy-Littlewood conjectures. The crucial point in his proof is that although $\mathfrak{S}(\mathcal{H}$ ) is not always 1 (as the Cramér model would have), it is approximately 1 on average over all $k$-element sets $\mathcal{H}$ with the $h_{j} \leq h$. That is, as $h \rightarrow \infty$,

$$
\sum_{1 \leq h_{1}<h_{2}<\ldots<h_{k} \leq h} \mathfrak{S}\left(\left\{h_{1}, \ldots, h_{k}\right\}\right) \sim \sum_{1 \leq h_{1}<h_{2}<\ldots<h_{k} \leq h} 1 .
$$

The ideas of Goldston, Pintz and Yıldırım. We will start with the idea behind Theorem 2. Let $k$ be a given positive integer which is at least 2. Let $\mathcal{H}=\left\{h_{1}<\right.$ $\left.\ldots<h_{k}\right\}$ be a set with $\mathfrak{S}(\mathcal{H}) \neq 0$. We aspire to the Hardy-Littlewood conjecture which says that there must be infinitely many $n$ such that $n+h_{1}, \ldots, n+h_{k}$ are all prime. Since there are infinitely many primes, trivially at least one of the numbers $n+h_{1}, \ldots, n+h_{k}$ is prime infinitely often. Can we do a little better: can we show 
that two of the numbers $n+h_{1}, \ldots, n+h_{k}$ are prime infinitely often? If we could, then we would plainly have that $\liminf _{p \rightarrow \infty}\left(p_{\text {next }}-p\right) \leq\left(h_{k}-h_{1}\right)$.

How do we detect two primes in $n+h_{1}, \ldots, n+h_{k}$ ? Let $x$ be large and consider $n$ varying between $x$ and $2 x$. Suppose we are able to find a function $a(n)$ which is always non-negative and such that, for each $j=1, \ldots, k$,

$$
\sum_{\substack{x \leq n \leq 2 x \\ n+h_{j} \text { prime }}} a(n)>\frac{1}{k} \sum_{x \leq n \leq 2 x} a(n) .
$$

Then summing over $j=1, \ldots, k$, it would follow that

$$
\sum_{x \leq n \leq 2 x} \#\left\{1 \leq j \leq k: n+h_{j} \text { prime }\right\} a(n)>\sum_{x \leq n \leq 2 x} a(n),
$$

so that for some number $n$ lying between $x$ and $2 x$ we must have at least two primes among $n+h_{1}, \ldots, n+h_{k}$.

Of course, the question is how do we find such a function $a(n)$ satisfying (4)? We would like to take $a(n)=1$ if $n+h_{1}, \ldots, n+h_{k}$ are all prime, and 0 otherwise. But then the problem of evaluating $\sum_{x \leq n \leq 2 x} a(n)$ is precisely that of establishing the Hardy-Littlewood conjecture.

The answer is suggested by sieve theory, especially the theory of Selberg's sieve. Sieve theory is concerned with finding primes, or numbers without too many prime factors, among various integer sequences. Some of the spectacular achievements of this theory are Chen's theorem [5] that for infinitely many primes $p$, the number $p+2$ has at most two prime factors; the result of Friedlander and Iwaniec [10] that there are infinitely many primes of the form $x^{2}+y^{4}$ where $x$ and $y$ are integers; and the result of Heath-Brown [19] that there are infinitely many primes of the form $x^{3}+2 y^{3}$ where $x$ and $y$ are integers. We recall here very briefly the idea behind Selberg's sieve.

Interlude on Selberg's sieve. We illustrate Selberg's sieve by giving an upper bound on the number of prime $k$-tuples $n+h_{1}, \ldots, n+h_{k}$ with $x \leq n \leq 2 x$. The idea is to find a 'nice' function $a(n)$ which equals 1 if $n+h_{1}, \ldots, n+h_{k}$ are all prime, and is non-negative otherwise. Then $\sum_{x \leq n \leq 2 x} a(n)$ provides an upper bound for the number of prime $k$-tuples. Of course, we must choose $a(n)$ appropriately so as to be able to evaluate $\sum_{x \leq n \leq 2 x} a(n)$.

Selberg's choice for $a(n)$ is as follows: Let $\lambda_{d}$ be a sequence of real numbers such that

$$
\lambda_{1}=1, \quad \text { and with } \quad \lambda_{d}=0 \quad \text { for } d>R .
$$

Choose 6

$$
a(n)=\left(\sum_{d \mid\left(n+h_{1}\right) \ldots\left(n+h_{k}\right)} \lambda_{d}\right)^{2} .
$$

Being a square, $a(n)$ is clearly non-negative. If $R<x \leq n$ and $n+h_{1}, \ldots, n+h_{k}$ are all prime, then the only non-zero term in (6) is for $d=1$ and so $a(n)=1$ as desired. Therefore we assume that $R<x$ below. The goal is to choose $\lambda_{d}$ so as to minimize $\sum_{x \leq n \leq 2 x} a(n)$. There is an advantage to allowing $R$ as large as possible, since this gives us greater flexibility in choosing the parameters $\lambda_{d}$. On the other

\footnotetext{
${ }^{6}$ The symbol $a \mid b$ means that $a$ divides $b$.
} 
hand it is easier to estimate $\sum_{x \leq n \leq 2 x} a(n)$ when $R$ is small since there are fewer divisors $d$ to consider. In the problem at hand, it turns out that we can choose $R$ roughly of size $\sqrt{x}$. This choice leads to an upper bound for the number of prime $k$-tuples of about $2^{k} \cdot k ! \mathfrak{S}(\mathcal{H}) x /(\log x)^{k}$. That is, a bound about $2^{k} \cdot k !$ times the conjectured Hardy-Littlewood asymptotic.

Expanding out the square in (6) and summing over $n$, we must evaluate

$$
\sum_{d_{1}, d_{2}} \lambda_{d_{1}} \lambda_{d_{2}} \sum_{\substack{x \leq n \leq 2 x \\ d_{1}\left|\left(n+h_{1}\right) \cdots\left(n+h_{k}\right) \\ d_{2}\right|\left(n+h_{1}\right) \cdots\left(n+h_{k}\right)}} 1=\sum_{d_{1}, d_{2}} \lambda_{d_{1}} \lambda_{d_{2}} \sum_{\substack{x \leq n \leq 2 x \\\left[d_{1}, d_{2}\right] \mid\left(n+h_{1}\right) \cdots\left(n+h_{k}\right)}} 1,
$$

where $\left[d_{1}, d_{2}\right]$ denotes the l.c.m. of $d_{1}$ and $d_{2}$. The condition $\left[d_{1}, d_{2}\right] \mid\left(n+h_{1}\right) \cdots$ $\left(n+h_{k}\right)$ means that $n$ must lie in a certain number (say, $\left.f\left(\left[d_{1}, d_{2}\right]\right)\right)$ of residue classes $\left(\bmod \left[d_{1}, d_{2}\right]\right)$. Can we count the number of $x \leq n \leq 2 x$ lying in the union of these arithmetic progressions? Divide the interval $[x, 2 x]$ into intervals of length $\left[d_{1}, d_{2}\right]$ with possibly one smaller interval left over at the end. Each complete interval (and there are about $x /\left[d_{1}, d_{2}\right]$ of these) gives $f\left(\left[d_{1}, d_{2}\right]\right)$ values of $n$; the last shorter interval contributes an indeterminate 'error' between 0 and $f\left(\left[d_{1}, d_{2}\right]\right)$. So, at least if $\left[d_{1}, d_{2}\right]$ is a bit smaller than $x$, we can estimate the sum over $n$ accurately. Since $\left[d_{1}, d_{2}\right] \leq d_{1} d_{2} \leq R^{2}$, if $R$ is a bit smaller than $\sqrt{x}$, then the sum over $n$ can be evaluated accurately. Let us suppose that $R$ is about size $\sqrt{x}$ and that the error terms can be disposed of satisfactorily. It remains to handle the main term contribution to $\sum_{x \leq n \leq 2 x} a(n)$, namely

$$
x \sum_{d_{1}, d_{2} \leq R} \frac{f\left(\left[d_{1}, d_{2}\right]\right)}{\left[d_{1}, d_{2}\right]} \lambda_{d_{1}} \lambda_{d_{2}} .
$$

The reader may wonder what $f\left(\left[d_{1}, d_{2}\right]\right)$ is. Let us work this out in the case when $\left[d_{1}, d_{2}\right]$ is not divisible by the square of any prime; the other case is more complicated but not very important in this problem. If $p$ is a prime and we want $p \mid(n+$ $\left.h_{1}\right) \cdots\left(n+h_{k}\right)$, then clearly $n \equiv-h_{j}(\bmod p)$ for some $j$, so that $n$ lies in one of $\nu_{\mathcal{H}}(p)$ residue classes $(\bmod p)$. By the Chinese remainder theorem it follows that if $\left[d_{1}, d_{2}\right] \mid\left(n+h_{1}\right) \cdots\left(n+h_{k}\right)$, then $n$ lies in $\prod_{p \mid\left[d_{1}, d_{2}\right]} \nu_{\mathcal{H}}(p)$ residue classes $\left(\bmod \left[d_{1}, d_{2}\right]\right)$. Thus $f$ is a multiplicative function 8 with $f(p)=\nu_{\mathcal{H}}(p)$.

The problem in Selberg's sieve is to choose $\lambda_{d}$ subject to the linear constraint (5) in such a way as to minimize the quadratic form (7) (that would give the best upper bound for $\left.\sum_{x \leq n \leq 2 x} a(n)\right)$. This can be achieved using Lagrange multipliers or by diagonalizing the quadratic form (7). We do not give the details of this calculation, but just record the result obtained. The optimal choice of $\lambda_{d}$ for $d \leq R$ is given by

$$
\lambda_{d} \approx \mu(d)\left(\frac{\log R / d}{\log R}\right)^{k}
$$

where $\mu(d)$ is the Möbius function 9 With this choice of $\lambda_{d}$ the quantity in (7) is

$$
\approx k ! \mathfrak{S}(\mathcal{H}) \frac{x}{(\log R)^{k}} \approx 2^{k} \cdot k ! \mathfrak{S}(\mathcal{H}) \frac{x}{(\log x)^{k}}
$$

\footnotetext{
${ }^{7}$ To be precise, $R$ must be $\leq \sqrt{x} /(\log x)^{2 k}$, say.

${ }^{8}$ These are functions satisfying $f(m n)=f(m) f(n)$ for any pair of coprime integers $m$ and $n$.

${ }^{9} \mu(d)=0$ if $d$ is divisible by the square of a prime. Otherwise $\mu(d)=(-1)^{\omega(d)}$ where $\omega(d)$ is the number of distinct primes dividing $d$.
} 
The appearance at this stage of the Möbius function is not surprising, as it is very intimately connected with primes. For example, the reader can check that $\sum_{d \mid m} \mu(d)(\log m / d)^{k}$ equals 0 unless $m$ is divisible by at most $k$ distinct prime factors. When $m=p_{1} \cdots p_{k}$ is the product of $k$ distinct prime factors, it equals $k !\left(\log p_{1}\right) \cdots\left(\log p_{k}\right)$, and there is a more complicated formula if $m$ is composed of fewer than $k$ primes or if $m$ is divisible by powers of primes. Applying this to $m=\left(n+h_{1}\right) \cdots\left(n+h_{k}\right)$, we are essentially picking out prime $k$-tuples! The optimum in Selberg's sieve is a kind of approximation to this identity.

Return to Goldston-Pintz-Yıldırım. We want to find a non-negative function $a(n)$ so that (4) will hold. Motivated by Selberg's sieve we may try to find optimal $\lambda_{d}$ as in (5) and again choose $a(n)$ as in (6). If we try such a choice, then our problem now is to maximize the ratio

$$
\left(\sum_{\substack{x \leq n \leq 2 x \\ n+h_{j} \text { prime }}} a(n)\right) /\left(\sum_{x \leq n \leq 2 x} a(n)\right) .
$$

We'd like this ratio to be $>1 / k$. Notice again that it is advantageous to choose $R$ as large as possible to give greatest freedom in choosing $\lambda_{d}$, but in order to evaluate the sums above there may be restrictions on the size of $R$. In dealing with the denominator we saw that there is a restriction $R \leq \sqrt{x}$ (essentially) and that in this situation the denominator in (8) is given by the quadratic form (7). We will see below that in dealing with the numerator of (8), a more stringent restriction on $R$ must be made: we can only take $R$ around size $x^{\frac{1}{4}}$.

In any case, (8) is the ratio of two quadratic forms, and this ratio needs to be maximized keeping in mind the linear constraint (5). This optimization problem is more delicate than the one in Selberg's sieve. It is not clear how to proceed most generally: Lagrange multipliers become quite messy, and we can't quite diagonalize both quadratic forms simultaneously. It helps to narrow the search to a special class of $\lambda_{d}$. Motivated by Selberg's sieve we will search for the optimum among the choices (for $d \leq R$ )

$$
\lambda_{d}=\mu(d) P\left(\frac{\log R / d}{\log R}\right) .
$$

Here $P(y)$ denotes a polynomial such that $P(1)=1$ and such that $P$ vanishes to order at least $k$ at $y=0$. The condition that $P$ be a polynomial can be relaxed a bit, but this is not important. It is, however, vital for the analysis that $P$ should vanish to order $k$ at 0 . Our aim is to find a choice for $P$ which makes the ratio in (8) large.

With this choice of $\lambda_{d}$ we can use standard arguments to evaluate (7) and thus the denominator in (8). Omitting the long, technical details, the answer is that for $R$ a little below $\sqrt{x}$, the denominator in $(8)$ is

$$
\sim \frac{x}{(\log R)^{k}} \mathfrak{S}(\mathcal{H}) \int_{0}^{1} \frac{y^{k-1}}{(k-1) !} P^{(k)}(1-y)^{2} d y,
$$

where $P^{(k)}$ denotes the $k$-th derivative of the polynomial $P$. 
To handle the numerator of (8), we expand out the square in (6) and sum over $x \leq n \leq 2 x$ with $n+h_{j}$ being prime. Thus the numerator is

$$
\sum_{d_{1}, d_{2} \leq R} \lambda_{d_{1}} \lambda_{d_{2}} \sum_{\substack{x \leq n \leq 2 x \\\left[d_{1}, d_{2}\right] \mid\left(n+h_{1}\right) \cdots\left(n+h_{k}\right) \\ n+h_{j} \text { prime }}} 1 .
$$

How can we evaluate the inner sum over $n$ ? As we saw before, the condition $\left[d_{1}, d_{2}\right]$ divides $\left(n+h_{1}\right) \cdots\left(n+h_{k}\right)$ means that $n$ lies in $f\left(\left[d_{1}, d_{2}\right]\right)$ arithmetic progressions $\left(\bmod \left[d_{1}, d_{2}\right]\right)$. For each of these progressions we must count the number of $n$ such that $n+h_{j}$ is prime. Of course, for some of the $f\left(\left[d_{1}, d_{2}\right]\right)$ progressions it may happen that $n+h_{j}$ automatically has a common factor with $\left[d_{1}, d_{2}\right]$ and so cannot be prime. Suppose there are $g\left(\left[d_{1}, d_{2}\right]\right)$ progressions such that $n+h_{j}$ is guaranteed to be coprime to $\left[d_{1}, d_{2}\right]$. For each of these progressions we are counting the number of primes between $x$ and $2 x$ lying in a reduced residue clas: $10 \quad\left(\bmod \left[d_{1}, d_{2}\right]\right)$. Given a modulus $q$, the prime number theorem in arithmetic progressions says that the primes are roughly equally divided among the reduced residue classes $(\bmod q)$. Thus, ignoring error terms completely, we expect the sum over $n$ to be about

$$
\frac{\pi(2 x)-\pi(x)}{\phi\left(\left[d_{1}, d_{2}\right]\right)} g\left(\left[d_{1}, d_{2}\right]\right) \text {. }
$$

The $\phi\left(\left[d_{1}, d_{2}\right]\right)$ in the denominator is Euler's $\phi$-function: for any integer $m, \phi(m)$ counts the number of reduced residue classes $(\bmod m)$. Since $\pi(2 x)-\pi(x)$ is about $x / \log x$ we 'conclude' that the numerator in (8) is about

$$
\frac{x}{\log x} \sum_{d_{1}, d_{2} \leq R} \lambda_{d_{1}} \lambda_{d_{2}} \frac{g\left(\left[d_{1}, d_{2}\right]\right)}{\phi\left(\left[d_{1}, d_{2}\right]\right)}
$$

This is the expression analogous to (7) for the numerator.

Two big questions: what is the function $g$, and for what range of $R$ can we handle the error terms above? Let us first describe $g$. As with $f$ let us suppose that $\left[d_{1}, d_{2}\right]$ is not divisible by the square of any prime. As noted earlier, if $p$ is prime and $p \mid\left(n+h_{1}\right) \cdots\left(n+h_{k}\right)$, then $n$ lies in one of $\nu_{\mathcal{H}}(p)$ residue classes $(\bmod p)$. If we want $n+h_{j}$ to be prime, then one of these residue classes, namely $n \equiv-h_{j}$ $(\bmod p)$, must be forbidden. Thus there are now $\nu_{\mathcal{H}}(p)-1$ residue classes available for $n(\bmod p)$. In other words, $g(p)=\nu_{\mathcal{H}}(p)-1$, and the Chinese remainder theorem shows that $g$ must be defined multiplicatively:

$$
g\left(\left[d_{1}, d_{2}\right]\right)=\prod_{p \mid\left[d_{1}, d_{2}\right]}\left(\nu_{\mathcal{H}}(p)-1\right) .
$$

We will postpone the detailed discussion on primes in arithmetic progressions which is needed to handle the error terms above. For the moment, let us note that the Bombieri-Vinogradov theorem (which is a powerful substitute for the generalized Riemann hypothesis in many applications) allows us to control $\pi(x ; q, a)$ (the number of primes up to $x$ which are congruent to $a(\bmod q)$ ), on average over $q$, for $q$ up to about $\sqrt{x}$. Since our moduli are $\left[d_{1}, d_{2}\right]$, which go up to $R^{2}$, we see that $R$ may be chosen up to about $x^{\frac{1}{4}}$. Conjectures of Montgomery, and Elliott and Halberstam (discussed below) would permit larger values of $R$, going up to $x^{\frac{1}{2}-\epsilon}$ for any $\epsilon>0$.

\footnotetext{
${ }^{10} \mathrm{~A}$ reduced residue class $(\bmod q)$ is a progression $a(\bmod q)$ where $a$ is coprime to $q$.
} 
Thus, with $R$ a little below $x^{\frac{1}{4}}$, the expression (10) does give a good approximation to the numerator of (8). Now a standard but technical argument can be used to evaluate (10). As with (9), the answer is

$$
\sim \frac{x}{(\log x)(\log R)^{k-1}} \mathfrak{S}(\mathcal{H}) \int_{0}^{1} \frac{y^{k-2}}{(k-2) !} P^{(k-1)}(1-y)^{2} d y .
$$

Assuming that $\mathfrak{S}(\mathcal{H}) \neq 0$, it follows from $(9)$ and $(11)$ that the ratio in (8) is about

$$
\frac{\log R}{\log x}\left(\int_{0}^{1} \frac{y^{k-2}}{(k-2) !} P^{(k-1)}(1-y)^{2} d y\right) /\left(\int_{0}^{1} \frac{y^{k-1}}{(k-1) !} P^{(k)}(1-y)^{2} d y\right) .
$$

This is the moment of truth: can we choose $P$ so as to make this a little larger than $1 / k ?$

Here is a good choice for $P$ : take $P(y)=y^{k+r}$ for a non-negative integer $r$ to be chosen optimally. After some calculations with beta-integrals, we see that (12) then equals

$$
\left(\frac{\log R}{\log x}\right)\left(\frac{2(2 r+1)}{(r+1)(k+2 r+1)}\right) .
$$

This is largest when $r$ is about $\sqrt{k} / 2$, and the second fraction above is close to but less than $4 / k$. Since we can choose $R$ a little below $x^{\frac{1}{4}}$, the first fraction is close to but less than $1 / 4$. Thus (12) is very close to, but less than, $1 / k$. We therefore barely fail to prove bounded gaps between primes! Of course, we just tried one choice of $P$; maybe there is a better choice which gets us over the edge. Unfortunately, the second fraction in (12) cannot be made larger than $4 / k$. If we set $Q(y)=P^{(k-1)}(y)$, then $Q$ is a polynomial, not identically zero, with $Q(0)=0$; for such polynomials $Q$ we claim that the unfortunate inequality

$$
\int_{0}^{1} \frac{y^{k-2}}{(k-2) !} Q(1-y)^{2} d y<\frac{4}{k} \int_{0}^{1} \frac{y^{k-1}}{(k-1) !} Q^{\prime}(1-y)^{2} d y
$$

holds. The reader can try her hand at proving this.

We now have enough to prove Theorem 2! If we can choose $R$ a little larger than $x^{\frac{1}{4}}$, then for suitably large $k$ the quantity in (12) can be made larger than $1 / k$ as desired. If we allow $R=x^{\frac{1}{2}-\epsilon}$ as the Elliott-Halberstam conjecture predicts, then with $k=7$ and $r=1$ we can make (12) nearly $1.05 / k>1 / k$. Thus, if we take any set $\mathcal{H}$ with seven elements and $\mathfrak{S}(\mathcal{H}) \neq 0$, then for infinitely many $n$ at least two of the numbers $n+h_{1}, \ldots, n+h_{k}$ are prime! By choosing a more careful polynomial $P$ we can make do with six element sets $\mathcal{H}$ rather than seven. The first six primes larger than 6 are $7,11,13,17,19$, and 23 , and so $\mathfrak{S}(\{7,11,13,17,19,23\}) \neq 0$. Thus, it follows that - assuming the Elliott-Halberstam conjecture - there are infinitely many gaps between primes that are at most 16 .

What can we recover unconditionally? We are so close to proving Theorem 2 unconditionally that clearly some tweaking of the argument must give Theorem 1 ! The idea here is to average over sets $\mathcal{H}$. For clarity, let us now denote $a(n)$ above by $a(n ; \mathcal{H})$ to exhibit the dependence on $\mathcal{H}$.

Given $\epsilon>0$ we wish to find primes $p$ between $x$ and $2 x$ such that $p_{\text {next }}-p \leq$ $\epsilon \log x$. This would prove Theorem 1 . Set $h=\epsilon \log x$, and let $k$ be a natural number chosen in terms of $\epsilon$ but fixed compared to $x$. Consider the following two sums:

$$
\sum_{1 \leq h_{1}<h_{2}<\ldots<h_{k} \leq h} \sum_{x \leq n \leq 2 x} a\left(n ;\left\{h_{1}, \ldots, h_{k}\right\}\right)
$$


and

$$
\sum_{1 \leq h_{1}<h_{2}<\ldots<h_{k} \leq h} \sum_{1 \leq \ell \leq h} \sum_{\substack{x \leq n \leq 2 x \\ n+\ell \text { prime }}} a\left(n ;\left\{h_{1}, \ldots, h_{k}\right\}\right) .
$$

If we could prove that (14) is larger than (13), it would follow that for some $n$ between $x$ and $2 x$, there are two prime numbers between $n+1$ and $n+h$, as desired.

Our analysis above already gives us the asymptotics for (13) and (14). Using (9) we see that the quantity (13) is

$$
\sim \frac{x}{(\log R)^{k}}\left(\int_{0}^{1} \frac{y^{k-1}}{(k-1) !} P^{(k)}(1-y)^{2} d y\right) \sum_{1 \leq h_{1}<h_{2}<\ldots<h_{k} \leq h} \mathfrak{S}\left(\left\{h_{1}, \ldots, h_{k}\right\}\right),
$$

and using Gallagher's result (3) this is

$$
\sim \frac{x}{(\log R)^{k}} \frac{h^{k}}{k !} \int_{0}^{1} \frac{y^{k-1}}{(k-1) !} P^{(k)}(1-y)^{2} d y .
$$

Now let us consider (14). Here we distinguish two cases: the case when $\ell=h_{j}$ for some $j$, and the case when $\ell \neq h_{j}$ for all $j$. The former case is handled by our analysis leading up to (11). Upon using (3) again, these terms contribute

$$
\sim k \frac{x}{(\log x)(\log R)^{k-1}} \frac{h^{k}}{k !} \int_{0}^{1} \frac{y^{k-2}}{(k-2) !} P^{(k-1)}(1-y)^{2} d y .
$$

If we choose $P(y)=y^{k+r}$ as before, we see that (16) is already just a shade below (15), so we need the slightest bit of extra help from the terms $\ell \neq h_{j}$ for any $j$. If $n+\ell$ is prime note that

$$
\begin{aligned}
a\left(n ;\left\{h_{1}, \ldots, h_{k}\right\}\right) & =\left(\sum_{d \mid\left(n+h_{1}\right) \cdots\left(n+h_{k}\right)} \lambda_{d}\right)^{2} \\
& =\left(\sum_{d \mid\left(n+h_{1}\right) \cdots\left(n+h_{k}\right)(n+\ell)} \lambda_{d}\right)^{2}=a\left(n ;\left\{h_{1}, \ldots, h_{k}, \ell\right\}\right),
\end{aligned}
$$

since the divisors counted in the latter sum but not the former are all larger than $n+\ell>x>R$ and so $\lambda_{d}=0$ for such divisors. This allows us to finesse the calculation by simply appealing to (11) again, with $k$ replaced by $k+1$ and $\left\{h_{1}, \ldots, h_{k}\right\}$ by $\left\{h_{1}, \ldots, h_{k}, \ell\right\}$. Thus the latter class of integers $\ell$ contributes

$$
\sim \sum_{1 \leq h_{1}<h_{2}<\ldots<h_{k} \leq h} \sum_{\substack{\ell=1 \\ \ell \neq h_{j}}}^{h} \frac{x}{(\log x)(\log R)^{k}} \mathfrak{S}\left(\left\{h_{1}, \ldots, h_{k}, \ell\right\}\right) \int_{0}^{1} \frac{y^{k-1}}{(k-1) !} P^{(k)}(1-y)^{2} d y .
$$

Appealing to (3) again - we are now summing over $k+1$ element sets but each set is counted $k+1$ times - this is

$$
\sim \frac{x}{(\log R)^{k}} \frac{h^{k}}{k !} \frac{h}{\log x} \int_{0}^{1} \frac{y^{k-1}}{(k-1) !} P^{(k)}(1-y)^{2} d y .
$$

This accounts for a factor of $\epsilon$ times the quantity in (15), and now the combined contribution of (16) and (17) may be made larger than (15), proving Theorem 1! 
Primes in arithmetic progressions. It remains to explain what is meant by the Bombieri-Vinogradov theorem and the Elliott-Halberstam conjecture. Recall that we required knowledge of these estimates for primes in progressions while discussing the error terms that arise while evaluating the numerator of (8).

Let us write

$$
\pi(x)=\operatorname{li}(x)+E(x)
$$

where $\operatorname{li}(x)$ stands for the 'logarithmic integral' $\int_{2}^{x} \frac{d t}{\log t}$, which is the expected main term, and $E(x)$ stands for an 'error term'. The main term li $(x)$ is, by integration by parts, roughly $x / \log x$. As for the error term $E(x)$, the standard proofs of the Prime Number Theorem give that for any number $A>0$ there exists a constant $C(A)$ such that

$$
|E(x)| \leq C(A) \frac{x}{(\log x)^{A}} .
$$

The argument generalizes readily for primes in progressions. Given an arithmetic progression $a(\bmod q)$ with $(a, q)=1$ let us write

$$
\pi(x ; q, a)=\frac{1}{\phi(q)} \operatorname{li}(x)+E(x ; q, a),
$$

where $\operatorname{li}(x) / \phi(q)$ is the expected main term - the primes are equally divided among the available residue classes - and $E(x ; q, a)$ is an 'error term' which we would like to be small. As with the Prime Number Theorem, for every $A>0$ there exists a constant $C(q, A)$ such that

$$
|E(x ; q, a)| \leq C(q, A) \frac{x}{(\log x)^{A}} .
$$

We emphasize that the constant $C(q, A)$ may depend on $q$. Therefore, this result is meaningful only if we think of $q$ as being fixed and let $x$ tend to $\infty$. In applications such a result is not very useful, because we may require $q$ not to be fixed, but to grow with $x$. For example, in our discussions above we want to deal with primes in progressions $\left(\bmod \left[d_{1}, d_{2}\right]\right)$ which can be as large as $R^{2}$, and we'd like this to be of size $x^{\frac{1}{2}}$ and would love to have it be even larger. Thus the key issue while discussing primes in arithmetic progressions is the uniformity in $q$ with which the asymptotic formula holds.

What is known about $\pi(x ; q, a)$ for an individual modulus $q$ is disturbingly weak. From a result of Siegel we know that for any given positive numbers $N$ and $A$, there exists a constant $c(N, A)$ such that if $q<(\log x)^{N}$, then

$$
|E(x ; q, a)| \leq c(N, A) \frac{x}{(\log x)^{A}} .
$$

This is better than the result for fixed $q$ mentioned earlier, but the range of $q$ is still very restrictive. An additional defect is that the constant $c(N, A)$ cannot be computed explicitly 11 in terms of $N$ and $A$.

If we assume the Generalized Riemann Hypothesis (GRH), then we would fare much better: if $x \geq q$ there exists a positive constant $C$ independent of $q$ such that

$$
|E(x ; q, a)| \leq C x^{\frac{1}{2}} \log x .
$$

This gives a good asymptotic formula for $\pi(x ; q, a)$ in the range $q \leq x^{\frac{1}{2}} /(\log x)^{3}$, say.

${ }^{11}$ This is not due to laziness, but is a fundamental defect of the method of proof. 
Given a modulus $q$ let us define

$$
E(x ; q)=\max _{(a, q)=1}|E(x ; q, a)| .
$$

We have discussed above the available weak bounds for $E(x ; q)$ and the unavailable strong GRH bound. Luckily, in many applications including ours, we don't need a bound for $E(x ; q)$ for each individual $q$, but only a bound holding in an average sense as $q$ varies. In the application to small gaps, we want primes in progressions $\left(\bmod \left[d_{1}, d_{2}\right]\right)$, but recall that we also have a sum over $d_{1}, d_{2}$ going up to $R$. An extremely powerful result of Bombieri and Vinogradov gives such an average estimate for $E(x ; q)$. Moreover, this average result is nearly as good as what would be implied by the GRH.

The Bombieri-Vinogradov theorem. For any positive constant $A$ there exist constants $B$ and $C$ such that

$$
\sum_{q \leq \mathcal{Q}} \max _{y \leq x}|E(y ; q)| \leq C \frac{x}{(\log x)^{A}},
$$

with $\mathcal{Q}=x^{\frac{1}{2}} /(\log x)^{B}$.

The constant $B$ can be computed explicitly; for example $B=24 A+46$ is permissible, but the constant $C$ here cannot be computed explicitly (a defect arising from Siegel's theorem mentioned above). The Bombieri-Vinogradov theorem tells us that on average over $q \leq \mathcal{Q}$ we have $E(x ; q) \leq C x(\log x)^{-A} / \mathcal{Q}=C x^{\frac{1}{2}}(\log x)^{B-A}$. Apart from the power of $\log x$, this is as good as the GRH bound!

A straightforward application of the Bombieri-Vinogradov theorem shows that as long as $R^{2} \leq x^{\frac{1}{2}} /(\log x)^{B}$ for suitably large $B$, the error terms arising in the Goldston-Pintz-Yıldırım argument will be manageable. If we wish to take $R$ larger, then we must extend the range of $\mathcal{Q}$ in (18). Such extensions are conjectured to hold, but unconditionally the range in (18) has never been improved upon 12

The Elliott-Halberstam conjecture. Given $\epsilon>0$ and $A>0$ there exists a constant $C$ such that

with $\mathcal{Q}=x^{1-\epsilon}$.

$$
\sum_{q \leq \mathcal{Q}} \max _{y \leq x}|E(x ; q)| \leq C \frac{x}{(\log x)^{A}},
$$

The Elliott-Halberstam conjecture would allow us to take $R=x^{\frac{1}{2}-\epsilon}$ in the Goldston-Pintz-Yıldırım argument. It is worth emphasizing that knowing (18) for $\mathcal{Q}=x^{\theta}$ with any $\theta>\frac{1}{2}$ would lead to a proof that there are bounded gaps between large primes.

Finally, let us mention a conjecture of Montgomery which lies deeper than the GRH and also implies the Elliott-Halberstam conjecture.

Montgomery's conjecture. For any $\epsilon>0$ there exists a constant $C(\epsilon)$ such that for all $q \leq x$ we have

$$
E(x ; q) \leq C(\epsilon) x^{\frac{1}{2}+\epsilon} q^{-\frac{1}{2}} .
$$

We have given a very rapid account of prime number theory. For more detailed accounts we refer the reader to the books of Bombieri [2, Davenport [6], and Montgomery and Vaughan [24.

\footnotetext{
${ }^{12}$ Although Bombieri, Friedlander and Iwaniec 4] have made important progress in related problems.
} 
Future directions. We conclude the article by mentioning a few questions related to the work of Goldston-Pintz-Yıldirım.

First and most importantly, is it possible to prove unconditionally the existence of bounded gaps between primes? As it stands, the answer appears to be no, but perhaps suitable variants of the method will succeed. There are other sieve methods available besides Selberg's. Does modifying one of these (e.g. the combinatorial sieve) lead to a better result? If instead of primes we consider numbers with exactly two prime factors, then Goldston, Graham, Pintz, and Yıldırım [13] have shown that there are infinitely many bounded gaps between such numbers.

In a related vein, assuming the Elliott-Halberstam conjecture, can one get to twin primes? Recall that under that assumption, we could show that infinitely many permissible 6-tuples contain two primes. Can the 6 here be reduced? Hopefully, to 2? Again the method in its present form cannot be pushed to yield twin primes, but maybe only one or two new ideas are needed.

Given any $\epsilon>0$, Theorem 1 shows that for infinitely many $n$ the interval $[n, n+\epsilon \log n]$ contains at least two primes. Can we show that such intervals sometimes contain three primes? Assuming the Elliott-Halberstam conjecture, one can get three primes in such intervals; see [12. Can this be made unconditional? What about $k$ primes in such intervals for larger $k$ ?

Is there a version of this method which can be adapted to give long gaps between primes? That is, can one attack Erdös's $\$ 10,000$ question?

\section{ACKNOWLEDGMENTS}

I am very grateful to Carine Apparicio, Bryden Cais, Brian Conrad, Sergey Fomin, Andrew Granville, Leo Goldmakher, Rizwan Khan, Jeff Lagarias, Youness Lamzouri, János Pintz, and Trevor Wooley for their careful reading of this article and for many valuable comments.

\section{ABOUt THE AUTHOR}

K. Soundararajan is currently a professor at Stanford University and the American Institute of Mathematics. He is a winner of the 2003 Salem Prize and the 2005 SASTRA Ramanujan Prize.

\section{REFERENCES}

[1] E. Bogomolny and J. Keating, Random matrix theory and the Riemann zeros. II. n-point correlations, Nonlinearity 9 (1996), 911-935. MR:1399479 (97d:11132b)

[2] E. Bombieri, Le grand crible dans la théorie analytique des nombres, vol. 18, Astérisque, 1987/1974. MR0891718 (88g:11064) MR0371840 (51:8057)

[3] E. Bombieri and H. Davenport, Small differences between prime numbers, Proc. Roy. Soc. Ser. A 293 (1966), 1-18. MR0199165 (33:7314)

[4] E. Bombieri, J. Friedlander and H. Iwaniec, Primes in arithmetic progressions to large moduli, Acta Math. 156 (1986), 203-251. MR0834613 (88b:11058)

[5] J.R. Chen, On the representation of a large even number as the sum of a prime and the product of at most two primes, Kexue Tongbao (Foreign Lang. Ed.) 17 (1966), 385-386. MR0207668 (34:7483)

[6] H. Davenport, Multiplicative number theory, Springer Verlag, 2000. MR:1790423 (2001f:11001)

[7] N. Elkies and C. McMullen, Gaps in $\sqrt{n}(\bmod 1)$ and ergodic theory, Duke Math. J. 123 (2004), 95-139. MR2060024 (2005f:11143)

[8] P. Erdős, On the difference of consecutive primes, Quart. J. Math. Oxford 6 (1935), 124-128. 
[9] P. Erdős, The difference between consecutive primes, Duke Math. J. 6 (1940), 438-441. MR0001759 (1:292h)

[10] J. Friedlander and H. Iwaniec, The polynomial $X^{2}+Y^{4}$ captures its primes, Ann. of Math. 148 (1998), 945-1040. MR.1670065 (2000c:11150a)

[11] P. X. Gallagher, On the distribution of primes in short intervals, Mathematika 23 (1976), 4-9. MR0409385(53:13140)

[12] D. Goldston, J. Pintz and C. Yıldırım, Primes in tuples, I, preprint, available at www. arxiv.org.

[13] D. Goldston, S. Graham, J. Pintz and C. Ylldırım, Small gaps between primes and almost primes, preprint, available at www.arxiv.org.

[14] D. Goldston, Y. Motohashi, J. Pintz and C. Yıldırım, Small gaps between primes exist, preprint, available at www.arxiv.org.

[15] A. Granville, Unexpected irregularities in the distribution of prime numbers, Proc. of the Int. Congr. of Math., Vols. 1, 2 (Zürich, 1994) (1995), 388-399. MR1403939 (97d:11139)

[16] G.H. Hardy and J.E. Littlewood, Some problems of Parititio Numerorum (III): On the expression of a number as a sum of primes, Acta Math. 44 (1922), 1-70.

[17] D.R. Heath-Brown, Prime twins and Siegel zeros, Proc. London Math. Soc. 47 (1983), 193224. MR0703977 (84m:10029)

[18] D.R. Heath-Brown, Differences between consecutive primes, Jahresber. Deutsch. Math.Verein. 90 (1988), 71-89. MR0939754 (89i:11100)

[19] D.R. Heath-Brown, Primes represented by $x^{3}+2 y^{3}$, Acta Math. 186 (2001), 1-84. MR1828372 (2002b:11122)

[20] M. Huxley, Small differences between consecutive primes. II, Mathematika 24 (1977), 142152. MR0466042 (57:5925)

[21] N. Katz and P. Sarnak, Zeros of zeta functions and symmetry, Bull. Amer. Math. Soc. 36 (1999), 1-26. MR1640151 (2000f:11114)

[22] H. Maier, Small differences between prime numbers, Michigan Math. J. 35 (1988), 323-344. MR0978303(90e:11126)

[23] H. Montgomery, The pair correlation of zeros of the zeta-function, Proc. Symp. Pure Math. 24 (St. Louis, MO, 1972), 181-193, Amer. Math. Soc., 1973. MR0337821 (49:2590)

[24] H. Montgomery and R.C. Vaughan, Multiplicative number theory I: Classical theory, Cambridge University Press, 2006.

[25] R. Rankin, The difference between consecutive primes, J. London Math. Soc. 13, 242-244.

[26] E. Szemeredi, On sets of integers containing no $k$ elements in arithmetic progression, Proc. International Congress of Math. (Vancouver) 2 (1975), 503-505. MR0422191 (54:10183)

[27] E. Westzynthius, Über die Verteilung der Zahlen, die zu der $n$ ersten Primzahlen teilerfremd sind, Comm. Phys. Math. Helsingfors 25 (1931), 1-37.

Current address: Department of Mathematics, Stanford University, 450 Serra Mall, Building 380, Stanford, California 94305-2125

E-mail address: ksound@math.stanford.edu

Department of Mathematics, University of Michigan, Ann Arbor, Michigan 48109

E-mail address: ksound@umich.edu 